\title{
INCIDENCIA DE LA DINÁMICA PLUVIOMÉTRICA EN LA DEGRADACIÓN DEL SUELO. SUR DE ESPAÑA
}

\author{
J.D. Ruiz Sinoga \\ Departamento de Geografía. Universidad de Málaga \\ sinoga@um.es \\ A. Romero Diaz \\ Departamento de Geografía. Universidad de Murcia \\ arodi@um.es \\ J.F. Martínez Murillo \\ Departamento de Geografía. Universidad de Málaga \\ jfmmurillo@uma.es \\ M.A. Gabarrón Galeote \\ Departamento de Geografía. Universidad Católica de Lovaina \\ miguel.gabarron@uclovain.es
}

\section{RESUMEN}

En la presente investigación: (i) se caracteriza el régimen de precipitaciones y la evolución pluviométrica durante el último medio siglo en las Cordilleras Béticas Litorales, sus tendencias y ciclos, (ii) se utiliza el método de reproducción de situaciones análogas en un gradiente pluviométrico, como una buena alternativa para el estudio comparado de procesos de degradación del suelo-desertificación en zonas próximas, con semejanzas en morfología, pero contraste climático; y (iii), se reflexiona acerca de posibles escenarios futuros, en el marco de la dinámica pluviométrica analizada.

Palabras clave: gradiente pluviomético, características eco-geomorfológicas, degradación del suelo, Sur de España.

\section{ABSTRACT}

In the present research have been: (i) characterized the regime of rainfall and rainfall evolution during the last half-century in the Bethic coastlines mountains, trends or cycles

Fecha de recepción: abril 2013.

Fecha de aceptación: mayo 2014. 
included, (ii) used the method of reproduction of similar situations in a rainfall gradient, as a good alternative to the comparative study of soil degradation and desertification processes in areas, with similarities in morphology, but contrast in climate; and (iii) established different future scenarios, in the framework of the analyzed pluviometric dynamics.

Keywords: rainfall gradient, Eco-geomorphological features. Soil degradation. Southern Spain.

\section{INTRODUCCIÓN}

En el sur de la Península Ibérica hay bien definido un gradiente pluviométrico, que oscila desde el clima mediterráneo húmedo al árido. A lo largo de este gradiente, un pequeño cambio es suficiente para modificar los límites entre estos dos ámbitos eco-geomorfológicos, de modo que muchas áreas mediterráneas situadas en el entorno de dicho umbral pueden ser sensibles a cambios en el patrón pluviométrico siendo amenazadas por procesos de degradación del suelo. Así, la posible incidencia del calentamiento global en el sistema eco-geomorfológico en determinados ambientes climáticos de transición, es una de las cuestiones que mayor expectativa está generando desde el punto de vista científico.

Dentro de los escenarios climáticos futuros planteados por el IPCC (2007 y 2013), la región mediterránea (Giorgi y Lionello, 2008) y especialmente el sur de España se configuran como una de las áreas de mayor incertidumbre, vinculada esta a la mayor o menor proximidad al Estrecho de Gibraltar y, por tanto, al factor mediterraneidad (De Castro et al., 2005; Sumner et al., 2003).

La regionalización de los dos principales escenarios de cambio climático llevada a cabo por la AEMET (Brunet et al., 2008), para el horizonte 2100 muestra, para Andalucía, un indudable incremento de las temperaturas máximas y mínimas, unido a una reducción de la pluviometría. Ambos escenarios mantienen similar tendencia aunque distinta intensidad y, en cualquier caso, muestran una amplia desviación estándar, es decir, una extraordinaria variabilidad. Similar tendencia encuentran Sánchez y Miguez-Macho para la Península Ibérica (2010).

El uso de series meteorológicas de precipitación y temperatura es de gran utilidad al aportar información sobre el comportamiento climático y sus repercusiones en los ecosistemas, mostrando como la conexión de los estudios climáticos con otros campos hace que se deriven amplias relaciones interdisciplinares.

En las últimas décadas han proliferado los estudios de la evolución de la precipitación, tanto a escala global como regional (Díaz et al., 1989; Amanatidis et al., 1993; Hulme, 1995; Brunetti et al., 2001; Norrant y Douguédroit, 2005; López-Bustins et al., 2008a y b; Mehta y Yang, 2008; entre otros). En el ámbito español también existen numerosos trabajos que tratan de analizar las variaciones y tendencias pluviométricas (Aguilar et al., 2006; Wheeler y Martín-Vide, 1992; Martin-Vide y Fernandez, 2001; Martin-Vide y Lopez-Bustins, 2006; Galán Gallego et al., 1999; González-Hidalgo et al., 2001, 2009; Saladié et al., 2002; Esteban-Parra et al, 2003; Ruiz Sinoga et al., 2011).

La preocupación existente por la evolución futura de los recursos hídricos en el contexto de la problemática del cambio climático ha suscitado un gran interés por el estudio de las 
tendencias de las series de precipitación. En el mediterráneo occidental, diversos autores (Moreno-García y Martín-Vide, 1986; De Luis et al., 2009) ya apreciaron una disminución de la precipitación en buena parte de la región, mientras que Quereda et al. (2000), en un análisis de 11 series largas, encontraron un aumento en Cataluña y Castellón, y una disminución en la restante vertiente mediterránea española.

Según el último informe del IPCC (2013), es probable que para finales del siglo XXI, la temperatura global en superficie sea superior en $1,5^{\circ} \mathrm{C}$ a la del período entre 1850 y 1900 para todos los escenarios considerados de trayectorias de concentración representativas (RCP), excepto para el escenario RCP 2,6. Es probable que esa temperatura sea superior en 2 ${ }^{\circ} \mathrm{C}$ para los escenarios RCP 6,0 y RCP 8,5, y más probable que improbable que sea superior en $2{ }^{\circ} \mathrm{C}$ para el escenario RCP 4,5. El calentamiento continuará después de 2100 en todos los escenarios RCP, excepto para el RCP 2,6. El calentamiento continuará mostrando una variabilidad entre interanual y decenal y no será uniforme entre las regiones.

La Cuenca Occidental del Mediterráneo, en el borde meridional de la zona templada, entre $\operatorname{los} 35^{\circ} \mathrm{N}$ y $\operatorname{los} 45^{\circ} \mathrm{N}$, podría estar abocada, de este modo, a experimentar impactos climáticos y biogeográficos de gran intensidad. En consecuencia, a lo largo del siglo XXI, se podría asistir a una sensible readaptación de su escenario biogeográfico afectado en gran medida por un aumento de la evapotranspiración y disminución de la precipitación, que según los modelos actuales (HadCM2, ECHAM4, Cosmo-CLM, PROMES model, etc.), se debería al progresivo alejamiento de la zona de generación del frente polar.

Los cambios que se producirán en el ciclo global del agua, en respuesta al calentamiento durante el siglo XXI, no serán uniformes. Se acentuará el contraste en las precipitaciones entre las regiones húmedas y secas y entre las estaciones húmedas y secas, si bien podrá haber excepciones regionales (IPCC, 2013). De manera general, se anticipa que la precipitación en la región Mediterránea disminuirá. Ello se debe, por un lado, a una tendencia generalizada a una reducción de la precipitación en los subtrópicos como resultado del incremento en la divergencia de vapor de agua (Held y Soden, 2006) y por otro a la migración hacia el norte del cinturón anticiclónico subtropical (Trenberth et al., 2007). También se ha argumentado que el calentamiento global podría conducir a un aumento de los eventos extremos de precipitación.

En conformidad con estas predicciones teóricas, las simulaciones del siglo XXI con modelos climáticos pronostican importantes disminuciones de la precipitación en todo el Mediterráneo, y en la Península Ibérica (PI) en particular, con especial incidencia en verano, donde el descenso podría alcanzar el 50\% a finales del siglo XXI (Meehl et al., 2007). La concordancia entre modelos es particularmente buena para esta región, lo cual aumenta la fiabilidad de esta proyección y señala a la región Mediterránea (ya de por sí caracterizada por condiciones semi-áridas) como un área particularmente vulnerable al calentamiento global.

No obstante, a lo largo del siglo XX, no se ha detectado una disminución generalizada y significativa de las precipitaciones en la región Mediterránea o en la PI (New et al., 2001; Norrant y Douguédroit, 2005). Una posible explicación es que, debido a la gran variabilidad temporal natural de la precipitación, la señal antropogénica esté tardando en emerger de forma evidente e inequívoca por encima del «ruido de fondo» natural de la precipitación. En tal caso, aún cuando exista una tendencia real, su detección y significación pueden depender 
en alto grado del periodo seleccionado y de los puntos inicial y final. Por otro lado, el sistema climático posee variabilidad natural a escalas de tiempo decenal y multidecenal, que puede manifestarse como oscilaciones de baja frecuencia, anomalías persistentes o tendencias pronunciadas durante dos o tres décadas (Giorgi y Lionello, 2008). Por ello es imperativo evaluar críticamente la significación estadística de las tendencias obtenidas. Esto requeriría disponer de series de precipitación largas, con periodos de referencia durante los cuales el forzamiento antropogénico pueda considerarse despreciable, pero este requisito no se cumple en la mayoría de los casos.

Para la Península Ibérica las precipitaciones medias estacionales simuladas reproducen de forma aceptable los gradientes norte-sur en verano y oeste-este en las demás estaciones del año. Los valores simulados de precipitaciones estacionales en la mitad sur peninsular española son generalmente menores que los climáticos reales (OECC, 2005).

Existen análisis pluviométricos que muestran determinadas tendencias para el sur de España (Ruiz Sinoga et al., 2011) que confirman los pronósticos ofrecidos a niveles regionales superiores por los distintos modelos de cambio climático mencionados, y cuáles pueden ser las repercusiones dentro del sistema eco-geomorfológico mediterráneo. Para ello, será necesario analizar los componentes bióticos, abióticos y antrópicos del geosistema, especialmente aquellos que sirven como indicadores de la salud y capacidad de respuesta del mismo ante el calentamiento global.

Las modificaciones producidas en las propiedades del suelo a lo largo de un gradiente pluviométrico pueden mostrar cómo una reducción de la precipitación anual podría suponer una menor cantidad de agua útil para las plantas, retrasando su germinación y reduciendo su actividad microbiológica, con lo que puede disminuir el contenido de materia orgánica al tiempo que se incrementaría la concentración de sales. Como consecuencia, podría disminuir el porcentaje de arcilla, afectando a la estabilidad estructural de los suelos, y modificando su capacidad de infiltración y permeabilidad. Esto supone un incremento en la tasa de escorrentía y flujo superficial, cuya consecuencia es la aparición de frecuentes costras superficiales o el inicio e intensificación de diferentes procesos de erosión hídrica, en un ciclo de retroalimentación (Lavee et al, 1998).

En el corto plazo, ha sido analizada la respuesta eco-geomorfológica de las variaciones pluviométricas en determinados ambientes mediterráneos del sur de España, plasmada en la modificación de algunas propiedades del suelo y en diferentes estrategias de adaptación de la comunidad vegetal (Ruiz Sinoga y Martinez Murillo, 2009). Además, las condiciones pluviométricas del ámbito de estudio y la humedad antecedente del suelo sobre el que se produce la precipitación, son factores que van a incidir directamente en las propiedades físicas e hidrológicas de las formaciones superficiales y en las características bióticas del medio (Imeson y Lavee, 1998). En definitiva, a lo largo de un gradiente pluviométrico y mediante la reproducción de situaciones análogas, como método predictivo, puede ser comprobado tal proceso (Bergkamp et al., 1999).

Bajo estos planteamientos, los objetivos del presente estudio han sido:

1. Analizar la evolución pluviométrica, sus tendencias y/o ciclos durante el último medio siglo en las Cordilleras Béticas Litorales, en las que existe un gradiente pluviométrico longitudinal. 
2. Comprobar en dicha área como una reducción dentro del gradiente pluviométrico incide en algunas propiedades edáficas, biológicas e hidrológicas y, por tanto, en el sistema eco-geomorfológico, pudiendo permitir la identificación de procesos de degradación del suelo, verificando si algunas de las propiedades edáficas analizadas pueden ser usadas como indicadoras de degradación del sistema.

3. Validar el método de reproducción de situaciones análogas en un gradiente pluviométrico, como una buena alternativa para el estudio comparado de procesos de degradación del suelo-desertificación en zonas próximas, con semejanzas en morfología, pero contraste climático, reflexionando sobre los posibles escenarios de futuro, en el supuesto de continuar la dinámica pluviométrica analizada.

Para conseguir estos objetivos, se ha analizado la evolución de la pluviometría en el sur de España, y las relaciones entre una serie de variables eco-geomorfológicas (edáficas, hidrológicas y biológicas) a lo largo de este gradiente, bajo diferentes regímenes de clima mediterráneo, como son: contenido de arcillas, limos y arenas, y arenas muy finas, materia orgánica, carbono orgánico retenido, salinidad, capacidad de intercambio catiónico, estabilidad estructural, factor «K» de la USLE, densidad aparente, conductividad hidráulica saturada (Ksat), humedad del suelo media, máxima y mínima, número de especies vegetales y cubierta vegetal.

La mayoría de estas propiedades son consideradas, en ambientes mediterráneos secos a semiáridos, como reguladoras de la erodibilidad del suelo (De Ploey y Poesen, 1985; Cerdá, 1998; Dunne et al., 1991), por su especial incidencia en: i) la vegetación, en ii) la humedad del suelo y en iii) el propio ciclo del carbono orgánico, siendo muchos los estudios que las han relacionado con los procesos de erosión del suelo (Le Bissonnais, 1996; Cammeraat y Imeson, 1998; Cerdá, 1998; Ruiz Sinoga y Romero Díaz, 2010).

i) Por un lado, porque las características de la vegetación varían a lo largo de gradientes climáticos (Whittaker, 1975; Ghazanfar, 1991; Ward y Olsvig-Whittaker, 1993; Shoshany et al., 1994, 1995; Kutiel et al., 1995; Kutiel et al., 2000). Numerosos estudios se han ocupado de las relaciones entre diversidad de vegetación y precipitación, y han concluido que no existe una manifiesta correlación entre diversidad de especies y precipitación, excepto cuando la precipitación supera los $400 \mathrm{~mm}$ anuales (Bowers, 1987; Aronson y Shmida, 1992; Ward y Olsvig-Whittaker, 1993), aunque sí puede encontrarse una clara correlación cuando se considera la distribución temporal de la precipitación (Bowers, 1987). Así pues, en la transición desde el clima mediterráneo húmedo al árido, el régimen pluviométrico es el factor limitante.

ii) En segundo lugar, porque el contenido de humedad de los suelos es la principal variable relacionada con la productividad de los suelos en áreas semiáridas (Le Houerou et al., 1988). La heterogeneidad espacial de este recurso es fundamental en el funcionamiento de estos ecosistemas y está íntimamente unido a la distribución espacial de los componentes bióticos y abióticos del paisaje (Noy-Meir, 1973), por lo que el análisis del contenido de humedad del suelo asociado a diversos tipos de cubierta vegetal dentro del paisaje, así como la distribución del agua, es importante para entender el funcionamiento del ecosistema (Tongway y Ludwig, 2003). 
iii) $\mathrm{Y}$ en tercer lugar, porque el incremento del contenido de humedad del suelo supone un aumento de los detritus derivados de la descomposición de la vegetación muerta y su incorporación como humus al suelo (Warren et al., 1986), lo que a su vez lo recarga de carbono orgánico, reduce la densidad aparente, mejora la estabilidad estructural (Thurow et al., 1986) e incrementa la conductividad hidráulica (Balliette et al., 1986). Así pues, comprender los cambios en el contenido de materia orgánica es no solo básico para determinar la calidad de los suelos (fertilidad, capacidad de infiltración...) y la productividad del ecosistema, sino también para cuantificar la influencia de los cambios en los ciclos del carbono orgánico y su almacenamiento en el contexto del cambio climático. Los ciclos de estos dos elementos juegan un papel esencial en la determinación de las características físicas y químicas de los suelos y, por tanto, en la determinación del nivel de degradación.

\section{II. ÁMBITO GEOGRÁFICO DE ESTUDIO}

El ámbito de estudio es el sur de España y en concreto las Cordilleras Béticas Litorales, en las que desde el punto de vista climático cabe distinguir entre clima subtropical, subdesértico, y de interior.

1. El clima mediterráneo subtropical caracteriza a la mayor parte de la costa mediterránea andaluza. La suavidad térmica invernal es su principal rasgo, y se debe a tres mecanismos básicos: la influencia suavizadora del mar, la orientación sur de la costa, que la convierte en una solana privilegiada para la recepción de los rayos solares, y la protección frente a las coladas septentrionales que le otorgan las cadenas montañosas Béticas. Las precipitaciones son muy variables e irregulares y, en general, muestran un gradiente de disminución oeste-este (Figura 1), desde los enclaves muy lluviosos del estrecho de Gibraltar hasta el dominio subdesértico de la costa almeriense (Capel, 1987).

2. El clima mediterráneo subdesértico caracteriza a todo el sector sur-oriental y se localiza en la provincia de Almería. Se trata de un área de muy escasas precipitaciones anuales, en la que las lluvias suelen ser inferiores a $200 \mathrm{~mm}$, y en algunos puntos cercanos al Cabo de Gata, incluso por debajo de los $150 \mathrm{~mm}$. Estas escasas precipitaciones se producen, además, en un número muy reducido de episodios de lluvias, muy intensas, lo que les otorga una fuerte torrencialidad, acentuando los procesos de erosión y degradación del suelo debido a la escasa cubierta vegetal existente, a lo que también contribuye la elevada evapotranspiración que se produce como consecuencia de las altas temperaturas y elevada insolación (Castillo, 1989).

3. El clima de interior y de montaña afecta a las áreas más elevadas, esencialmente Trevelez, pero también todas las altas sierras que la rodean, así como los enclaves más altos de la Serranía de Ronda. El efecto general de la altitud se plasma en una reducción térmica, tanto mayor cuanto más elevado sea el lugar, y en un aumento pluviométrico, muy condicionado por la posición topográfica y los relieves circundantes (en Gaucín, al suroeste de la Serranía de Ronda, se superan los $1.100 \mathrm{~mm}$ de lluvia media anual). A pesar del efecto del factor altitudinal, su ubicación en la fachada 
meridional, hacia el sur y la proximidad del mar, muestra una curva ombrotérmica con unas características mediterráneas, siendo la sequía estival tan acusada como en el resto de la región, por lo que el efecto orográfico no puede imponerse a la marcada subsidencia del aire que se deriva de la presencia de las altas presiones subtropicales. No obstante, la sequía estival, unida a los rigores térmicos invernales, configura unas condiciones de extraordinaria dureza tanto para la vegetación y la fauna como para la ocupación humana (Pita, 2003).

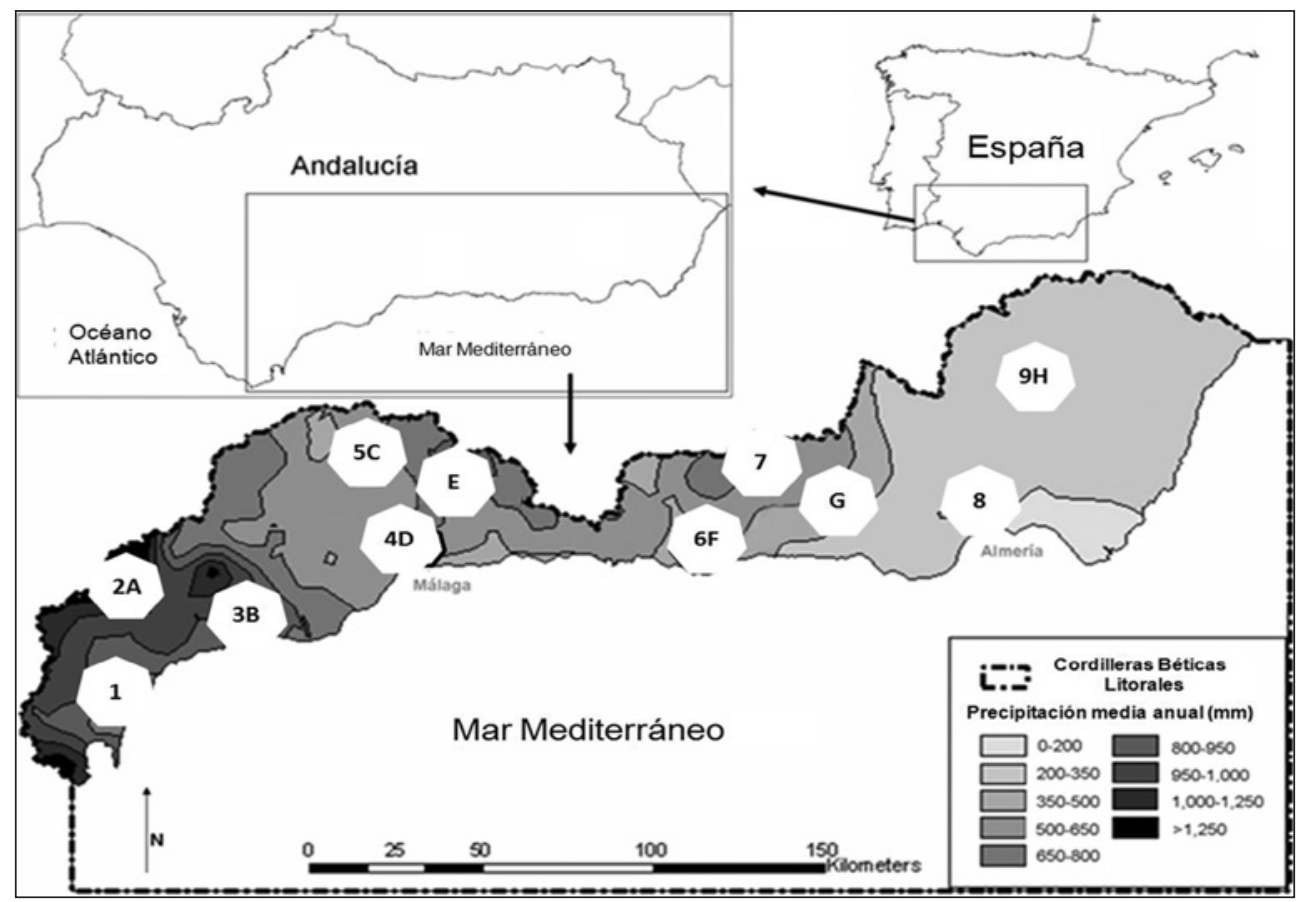

\section{MATERIALES Y MÉTODOS}

\section{III.1. Determinación del gradiente y dinámica pluviométrica}

El gradiente climático observado en el área de estudio fue definido mediante el análisis de una serie de datos pluviométricos diarios (1960-2006) que incluía un total de 271 estaciones pluviométricas distribuidas desde el Estrecho de Gibraltar hasta Almería, a lo largo de la Cuenca Mediterránea Andaluza, perteneciente a la antigua Confederación Hidrográfica del 
Sur de España, hoy Agencia Andaluza del Agua. El mapa del gradiente de precipitación fue obtenido mediante técnicas geoestadísticas de kriging y ajustando un modelo esférico a la distribución de puntos. Sin embargo, al analizar la dinámica pluviométrica del último medio siglo, a pesar de disponer de una adecuada representatividad espacial de los observatorios (271), para comprobar el comportamiento y evolución de las precipitaciones, algunos presentan discontinuidades, lagunas o simplemente series cortas, por lo que en total solo se han considerado 196 observatorios.

En los observatorios seleccionados (Tabla 1), (Almería, Motril, Málaga, Marbella, San Roque, Gérgal, Trevelez, Antequera, Gaucín) algunas lagunas de información se han cubierto mediante interpolación, homogeneizando la base de datos de precipitación de dichos observatorios; para obtener datos uniformes de calidad, poniendo a prueba la homogeneidad de los registros como paso previo y preciso en cualquier análisis climático. Para la detección y corrección de posibles errores se ha aplicado el Standard normal Homogeneity Test (SNHT), definido por Alexanderson (1986) y modificado después por Alexanderson y Moberg (1997), conforme a lo desarrollado por Ruiz Sinoga et al., (2011).

Para establecer o deducir un pronóstico evolutivo de la precipitación para los años siguientes al último de la serie, es decir para después de 2007, debe sustituirse la línea poligonal por otra prolongable que se adapte o ajuste de la mejor forma posible a ella. Conviene, pues, sustituir los puntos verdaderos representativos de las lluvias reales por otros ficticios que estén en una línea de ley de distribución conocida (recta, parábola, etc.) y cuya distancia residual a los respectivos valores reales sea la mínima posible. En este caso, los métodos empleados para el cálculo de la trayectoria de la precipitación son el método de la tendencia definido por regresión lineal, y las medias móviles con banda de 5 años. Gracias a estos promedios móviles se crea una nueva serie, creciente o decreciente, que permite deducir una determinada tendencia secular en la serie estudiada (Brightman, 1999; Walford, 1995). Para evaluar la significación estadística de las tendencias obtenidas se ha utilizado el test no paramétrico de Mann Kendall (Z-test) a un nivel de confianza del 95\%.

Tabla 1

PRINCIPALES CARACTERÍSTICAS DE LAS ESTACIONES METEOROLÓGICAS ANALIZADAS

\begin{tabular}{|c|c|c|c|}
\hline Observatorio & Coordenadas & Altitud $(\mathrm{m})$ & Serie \\
\hline Almería & $4077256 \mathrm{~N}-547479 \mathrm{~W}$ & 6 & $1961-2007$ \\
\hline Málaga & $4064690 \mathrm{~N}-373188 \mathrm{~W}$ & 12 & $1961-2007$ \\
\hline Motril & $4067264 \mathrm{~N}-453879 \mathrm{~W}$ & 45 & $1961-2007$ \\
\hline San Roque & $4009846 \mathrm{~N}-285647 \mathrm{~W}$ & 109 & $1961-2007$ \\
\hline Marbella & $4041057 \mathrm{~N}-331329 \mathrm{~W}$ & 115 & $1961-2007$ \\
\hline Antequera & $4097869 \mathrm{~N}-360630 \mathrm{~W}$ & 540 & $1961-2007$ \\
\hline Gaucín & $4043753 \mathrm{~N}-292558 \mathrm{~W}$ & 697 & $1961-2007$ \\
\hline Gérgal & $4131968 \mathrm{~N}-544293 \mathrm{~W}$ & 886 & $1961-2007$ \\
\hline Trevelez & $4091190 \mathrm{~N}-483681 \mathrm{~W}$ & 1319 & $1961-2007$ \\
\hline
\end{tabular}




\section{III.2. Análisis de la dinámica y tendencia de las rachas secas}

Se ha determinado la evolución de los periodos secos usando el índice DDSLR (Dry Days Since Last Rain), con el fin de caracterizar las tendencias del numero de días secos a lo largo de un gradiente pluviométrico, determinar como el DDSLR cambia a lo largo del gradiente desde las áreas mediterráneas semiáridas a las húmedas $\mathrm{y}$, por tanto, valorar su contribución en la dinámica suelo-agua-vegetación (Ruiz Sinoga et al., 2012). El método DDSLR (Aviad et al., 2009; Reiser and Kutiel, 2010) permite el cálculo de la probabilidad de la ocurrencia de días secos (días sin lluvia) en cualquier momento del año, evaluando el riesgo de déficit de agua para la vegetación durante el periodo activo, y determinando la variabilidad temporal de los procesos hidrológicos/geomorfológicos que dependen de la humedad del suelo durante todo el año. Por ejemplo, la posibilidad de ampliación de la estación seca a los meses de septiembre y octubre se puede convertir en un periodo critico con severas implicaciones para la disponibilidad de los recursos hídricos del suelo para la vegetación.

Reiser y Kutiel (2010) dividieron el Mediterráneo en cuatro regiones: (1) la región meridional, que tiene máxima aridez en agosto y septiembre; (2) al noreste de la Península Ibérica, sur de Francia y el norte-oeste de Italia, que es menos árido de la región 1 y tiene un déficit de agua en julio y agosto; (3) al noreste del Mediterráneo Oriental, que tiene dos períodos de sequía moderada durante el año (la más severo desde agosto a octubre y otro de enero a marzo); y (4) el área del noroeste del Mediterráneo Oriental, que también tiene sequía dos periodos cada año (la más severa desde enero a marzo y otra en octubre). Según esta clasificación el sur del Mediterráneo español está incluido en la región 1, pero tiene sequía hasta octubre.

Un día seco se define como aquel durante el que no hay ninguna precipitación o la cantidad de lluvia está por debajo de un umbral predeterminado. Los umbrales han variado según los objetivos y métodos utilizados en particular por los diferentes estudios. El umbral más comúnmente usado $(0,1 \mathrm{~mm})$ representa la precisión típica de un pluviómetro (Berger y Goossens, 1983; Martín al vacío y Gómez, 1999; Ceballos et al., 2004). Otros umbrales utilizados han estado relacionados con la definición de 'lluvia significativa', que generalmente se determina por evapotranspiración diaria. Los valores típicos son 1,0 mm (Jackson, 1981; Douguedroit, 1987), 2,0 mm (Perzyna, 1994), 5,0 mm (Cook y Heerdegen, 2001) y $30 \mathrm{~mm}$. En este estudio, hemos definido cuatro umbrales de precipitación diaria (DRTs) debido a su potencial para afectar a diversos procesos hidrológicos, geomorfológicos y ecológicos. El primer DRT $(0,1 \mathrm{~mm})$ es la cantidad diaria mínima registrada por los pluviómetros de la Agencia Meteorológica estatal de España (AEMET). Después de un verano largo seco típico del área mediterránea, el contenido de agua del suelo es muy bajo (menos del punto de marchitamiento), y el suelo se convierte en hidrofóbico, reduciendo su capacidad de infiltración, incluso cuando las características superficiales (numerosos fragmentos de roca en la superficie del suelo) favorecen los procesos de infiltración (Ruiz Sinoga y Martínez Murillo, 2009).

Esto favorece la escorrentía cuando las precipitaciones son inferiores a 10,0 mm durante la primera parte de la temporada hidrodinámica (septiembre a diciembre) (Martínez Murillo y Ruiz Sinoga, 2007), y por esta razón hemos determinado el segundo DRT (5.0 mm). El 
tercer DRT (10,0 $\mathrm{mm})$ refleja la precipitación mínima que tiene efectos sobre el contenido de humedad edáfica a profundidades mayores a $5 \mathrm{~cm}$ (Ceballos et al., 2002) y genera escorrentía cuando la humedad del suelo es insignificante o nula (Yair y Lavee, 1985; Ceballos y Schnabel, 1998). La cuarta DRT (30 mm) corresponde a la cantidad de lluvia que provoca efectos fenológicos en vegetación en condiciones del desierto (Beatley, 1974; Veenendaal et al.,1996), y produce flujos escorrentía lineal de corta duración (3 h o menos) (Ceballos y Schnabel, 1998).

\section{III.3. Selección de las áreas de estudio y características eco-geomorfológicas}

A lo largo de las Cordilleras Béticas Litorales se han seleccionado diferentes áreas representativas, tanto desde el punto de vista pluviométrico como eco-geomorfológico. Por una parte, se ha analizado la serie de los registros pluviométricos diarios de 9 observatorios meteorológicos, 5 localizados a lo largo del litoral y otros 4 en el tras país interior (Figura 1 y Tabla 1). Por otra, se han estudiado 8 laderas representativas que comparten rasgos topográficos (orientación sur y perfil convexo con aumento de la pendiente hacia la base o depresión), geológicos (sustrato geológico de rocas metamórficas) y de uso del suelo (condiciones casi-naturales con pastoreo no intensivo). Las diferencias están relacionadas con: el clima, cambios en la composición y el patrón de la vegetación, propiedades de los suelos y procesos geomorfológicos. Desde Gaucín (GA) hasta Gérgal (GE) existe una importante reducción de las precipitaciones y, por tanto, de la cubierta vegetal, apareciendo especies vegetales cada vez más adaptadas a la falta de agua (Figura 2).

Figura 2

ASPECTO DE LA CUBIERTA VEGETAL EN LAS DIFERENTES ÁREAS DE ESTUDIO

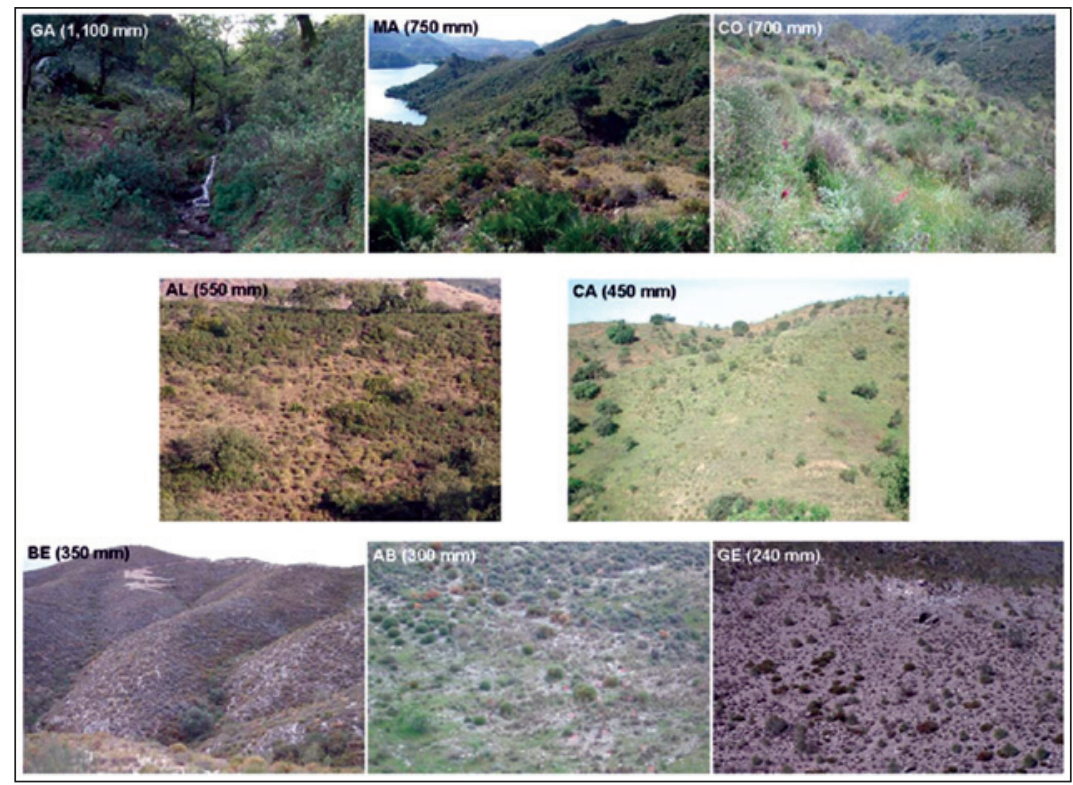




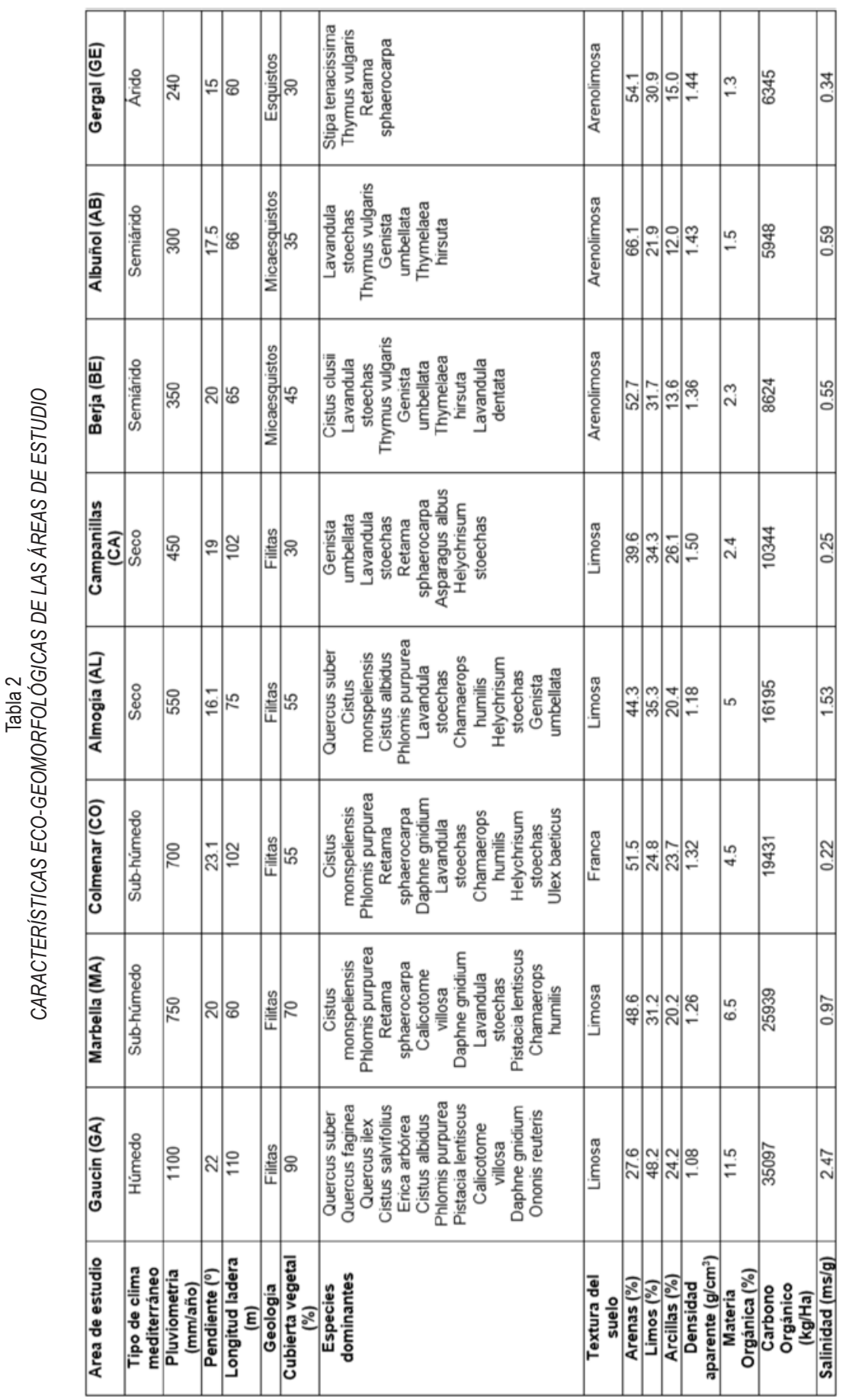




\section{III.4. Análisis del sistema eco-geomorfológico}

La metodología empleada utiliza un gradiente pluviométrico mediterráneo (Imeson y Lavee, 1998), para lo que se seleccionan varias áreas de estudio representativas (laderas) mediante la aplicación de unos criterios de semejanzas (topografía, geología y uso del suelo) y diferencias (relacionadas con las condiciones climáticas).

Se tomaron un total de 469 muestras superficiales del suelo (0-5 cm): 90 en Gaucín (GA), 40 en Marbella (MA), 52 en Colmenar (CO), 60 en Almogía (AL), 63 en Campanillas (CA), 50 en Berja (BE), 54 en Albuñol (AB) y 60 en Gérgal (GE). El número de muestras totales por área de estudio varió según la superficie a muestrear en las laderas. Las muestras se tomaron tanto alteradas como inalteradas en cilindros de $100 \mathrm{~cm}^{3}$ (Tabla 2).

Las propiedades del suelo analizadas con las muestras alteradas fueron: textura, materia orgánica $(\mathrm{MO})$, densidad aparente (DA), conductividad eléctrica (CE) y capacidad de intercambio catiónico (CIC). La textura fue analizada mediante el método de Robinson (Robinson, 1922). La MO fue analizada mediante el método AFNOR de medida del carbono (AFNOR, 1987). La CE fue medida por una solución acuosa 1:1 (w/v). La muestras inalteradas en cilindros de $100 \mathrm{~cm}^{3}$ permitieron analizar la DA la cual fue determinada por el «core method» (Blake y Hartge, 1986). La CIC fue determinada mediante saturación de bases intercambiables con el índice del catión (NH4+) 1 N NH4OAc a pH 8.2 (Bower et al., 1952). El carbono orgánico almacenado en el suelo (COS) para los primeros $10 \mathrm{~cm}$ de suelo fue calculado para cada área de estudio como sigue:

$$
\operatorname{COS}\left(\mathrm{kg} \mathrm{ha}^{-1}\right)=(\% \mathrm{COS} / 100) \times \text { Agregación del suelo }\left(\mathrm{kg} \mathrm{ha}^{-1}\right)
$$

donde la «agregación del suelo» $\left(\mathrm{kg} \mathrm{ha}^{-1}\right)=$ profundidad $(\mathrm{m}) \times \mathrm{DA}\left(\mathrm{M} \mathrm{g} \mathrm{m}^{-3}\right) \times 10,000 \mathrm{~m}^{2} \mathrm{ha}^{-1}$ x $1000 \mathrm{~kg} \mathrm{Mg}^{-1}$.

La erodibilidad del suelo (USLE K factor) fue calculada de acuerdo con Wischmeier y Smith (1978) para cada área de estudio de la siguiente forma:

$$
\mathrm{K}=2.8 \mathrm{M}^{1.14}\left(10^{-7}\right)(12-\mathrm{MO})+4.3\left(10^{-3}\right)(\mathrm{S}-2)+3.3\left(10^{-3}\right)(\mathrm{P}-3)
$$

donde $\mathrm{M}$ es el parámetro referido al tamaño de las partículas calculado como (\% de limos,+ $\%$ de arenas finas) (100- \% de arcillas), MO la Materia Orgánica (\%); S código de estructura del suelo (1=grano muy fino, 2=grano fino, 3= grano medio o grueso, 4=estructura masiva); y P la permeabilidad del suelo (1=rápida, $2=$ =moderada a rápida, $3=$ moderada, $4=$ baja a moderada, $5=$ baja, $6=$ muy baja) (Tabla 2 ).

\section{III.5. Análisis estadístico}

A la base de datos se le aplicó el coeficiente de correlación de Pearson para evaluar el grado de correlación existente entre las variables analizadas. Asimismo, se aplicaron los test de Bartlett y KMO (Kaiser-Meyer-Olkin) para evaluar la posibilidad de aplicar un análisis factorial (en adelante ACP), el cual se llevó a cabo para determinar agrupaciones entre variables. Este análisis factorial se ejecutó usando la matriz de covarianza (datos brutos) y 
la de correlaciones (datos estandarizados), mediante la utilización del programa estadístico SPSS-15 (Chicago, USA). Mediante el uso de la matriz de correlaciones, los factores con «eigenvalues» >1 fueron mantenidos y sometidos a la rotación «Varimax» para maximizar su nivel de correlación.

\section{RESULTADOS Y DISCUSIÓN}

\section{IV.1. La desigual dinámica pluviométrica}

A pesar de que la irregularidad pluviométrica interanual es el patrón temporal general, existen diferencias apreciables, dependiendo de la localización geográfica de los observatorios, en cuanto al número y duración de ciclos inusualmente húmedos o secos. El litoral oriental (Almería) presenta un menor número de ciclos húmedos que los restantes observatorios analizados (Ruiz Sinoga et al., 2011). Para todo el periodo de análisis se observan 4 ciclos húmedos bien definidos, cuya duración apenas si supera los 4 años, siendo los secos, más prolongados, de hasta 10 años (1978-1987), aunque aquellos sean más intensos. Hacia el oeste los ciclos húmedos presentan una duración similar a los secos y, además, los ciclos secos están peor definidos, lo que supone una tendencia pluviométrica positiva (Marbella y San Roque). Los observatorios del interior presentan unos resultados mucho más significativos (Gérgal, Trevelez, Antequera y Gaucín). El número de ciclos húmedos es similar al de los observatorios del litoral mediterráneo suroccidental, aunque poseen menor intensidad, de ahí la tendencia decreciente de la precipitación anual (Figura 1).

Para el periodo analizado, y de acuerdo con Quereda et al. (2000), puede considerarse que la evolución pluviométrica regional muestra buena correspondencia con la actividad solar. Los ciclos pluviométricos y los ciclos solares presentan un aceptable paralelismo, de manera que durante periodos de gran número de manchas solares se manifiestan los ciclos húmedos y al contrario. No obstante, esta correlación no es perfecta. Así, 1980, año correspondiente a un ciclo de máxima actividad solar, coincide con un mínimo pluviométrico. Una explicación satisfactoria a esta contrariedad puede encontrarse en el efecto de la actividad volcánica de los volcanes Santa Helena (1980) y Chinchón (1982), cuyos aerosoles pudieron bloquear la mayor radiación solar y su potenciación de mayores precipitaciones (Quereda et al., 2000).

Estas correlaciones positivas entre mayor o menor radiación solar y ciclos húmedos o secos vuelven a recobrarse durante los años lluviosos de 1989-1990 y el periodo de sequía intensa que irrumpió durante los años 1994-1995. Durante los últimos años de la década de los años 90 del pasado siglo XX y primeros años del nuevo milenio, un nuevo periodo de máxima actividad solar se relaciona con un nuevo ciclo húmedo; mientras que desde el año 2004 y hasta el 2007 un reciente periodo de sequía se corresponde con un descenso en el número de manchas solares. En la tabla 3 se describe la tendencia observada y su incidencia anual. En este caso se vuelven a advertir diferencias notables derivadas de la localización del observatorio.

En el litoral oriental existe una tendencia negativa, hacia una menor precipitación total anual (Almería y Motril). Incluso el observatorio de Málaga, localizado en una posición central respecto al mediterráneo sur español, podría unirse a este grupo. 
En el litoral occidental, Marbella y San Roque, se aprecian unas tendencias positivas, hacia una mayor precipitación total anual, con unos incrementos pluviométricos anuales de 2,7 y $2,2 \mathrm{~mm} / \mathrm{año}$ respectivamente.

En el interior puede observarse también una tendencia negativa, con una reducción de la pluviometría anual considerable (Trevelez, Antequera y Gaucín).

Las tendencias de precipitación son menos intensas en la costa sur mediterránea oriental (Almería, Motril, Gérgal), independientemente de su posición interior o litoral, respecto a los observatorios occidentales (Marbella, San Roque, Gaucín). La significación estadística de estas tendencias se ha valorado mediante la aplicación del test Mann-Kendall. Este test ha demostrado que solamente los observatorios del interior mediterráneo andaluz (Trevelez, Antequera y Gaucín) ofrecen una tendencia negativa significativa (Tabla 3). La única excepción la constituye el observatorio de Gérgal, cuya tendencia negativa no ofrece una significación estadística elevada. Según Sumner et al. (2003), existe una marcada disminución de los flujos húmedos que provienen del oeste, atlánticos, situaciones que provocan en el sur mediterráneo español la mayor cantidad de precipitaciones en invierno y primavera. Como consecuencia, la cantidad de precipitación total anual tiende a reducirse.

Tabla 3

TENDENCIAS DE LA PRECIPITACIÓN ANUAL (1961-2007). REDUCCIÓN O AUMENTO DE LA PRECIPITACIÓN (MM / AÑO)

\begin{tabular}{|c|c|c|c|c|c|}
\hline Observatorio & Invierno & Primavera & Verano & Otoño & Anual \\
\hline Almería & -0.34 & -0.40 & +0.30 & -0.11 & -0.55 \\
\hline Motril & -1.72 & -0.44 & +0.28 & +1.67 & -0.21 \\
\hline Málaga & -0.70 & +0.17 & +0.15 & -0.19 & -0.57 \\
\hline Marbella & -1.22 & +0.67 & +0.72 & +2.56 & +2.73 \\
\hline San Roque & -0.79 & -0.23 & +0.45 & +2.79 & +2.22 \\
\hline Gérgal & -0.54 & -0.22 & +0.22 & -0.98 & -1.52 \\
\hline Trevelez & -2.02 & -0.70 & +0.47 & -1.08 & -3.33 \\
\hline Antequera & -1.51 & -0.23 & +0.11 & -1.49 & -3.12 \\
\hline Gaucín & -5.83 & +0.96 & +0.59 & -0.39 & -4.67 \\
\hline
\end{tabular}

Conocida la tendencia pluviométrica anual, se ha analizado la evolución estacional de la precipitación, pues es esta la que una mayor incidencia puede ejercer en el periodo activo o de crecimiento de la vegetación y los cultivos. Así, y a modo de ejemplo, una tendencia pluviométrica negativa y una prolongación de episodios secos durante la primavera u otoño, épocas clave para el desarrollo vegetativo, podría acarrear consecuencias directas sobre el estado de salud sistema eco-geomorfológico.

En términos globales, la tendencia de la precipitación invernal (1961-2007) muestra una trayectoria decreciente generalizada. Durante los equinoccios, las tendencias son variables, sin embargo se advierten ciertas similitudes según las distintas zonas geográficas de análisis 
(Tabla 3). En primavera se observa un doble patrón en la tendencia pluviométrica. En aquellos observatorios más orientales, ya sean litorales o de interior, la precipitación de primavera desciende, mientras que los suroccidentales ofrecen una tendencia positiva, a excepción de San Roque, aunque la tendencia negativa no es muy significativa. Durante el otoño, aparecen tres patrones distintos en las tendencias pluviométricas. En el interior (Gérgal, Trevelez, Antequera y Gaucín) y en el litoral oriental más oriental (Almería) la tendencia es negativa, si bien menos significativa en este último caso. Los observatorios del litoral centro-oriental (Motril y Málaga) poseen una tendencia positiva. Y los observatorios occidentales (Marbella y San Roque) presentan una tendencia positiva y de incremento nítido en la precipitación de otoño. En verano, el análisis de la tendencia pluviométrica muestra signos positivos en todos los observatorios analizados.

En otros estudios, sobre las tendencias de precipitación en la Península Ibérica se encuentran ciertas similitudes con el análisis aquí realizado. Según López-Bustins et al. (2008), la mitad occidental del sur mediterráneo español presenta tendencias decrecientes, sobre todo la precipitación invernal. Además de analizar su dependencia respecto a la variación de la NAO (Oscilación del Atlántico Norte), los autores citados piensan que este descenso de la precipitación se relaciona con una situación sinóptica típica del periodo central del invierno: potente anticiclón centrado sobre Europa Central, comportando estabilidad atmosférica sobre el centro y oeste de la Península Ibérica y una circulación del NE en la fachada oriental mediterránea. Este patrón ha incrementando su frecuencia del orden de 2,71 días/década en los meses centrales del invierno a lo largo del periodo 1959-2000. Esto significa un aumento de la presión atmosférica en la planicie centroeuropea en invierno, como corroboran varios estudios previos (Stefanicki et al., 1998; Maugeri et al., 2004; Trigo, 2006). Este patrón de circulación atmosférica también se corresponde con una fase típicamente positiva de la NAO, lo cual justifica la reducción pluviométrica.

De acuerdo con Guijarro (2002), la elevada variabilidad espacio-temporal de la precipitación estacional, junto a la escasa significación estadística a veces encontrada (fruto de la elevada variabilidad de la propia precipitación), puede llevar a menospreciar la importancia de las tendencias pluviométricas señaladas, sin embargo, de mantenerse en el tiempo, supondrían cambios drásticos en la disponibilidad de agua por parte del ecosistema, lo que repercutiría directamente en la gestión y el consumo del agua por parte de la vegetación, y consecuentemente, en estrategias de adaptación.

\section{IV.2. Incidencia de las rachas secas}

La probabilidad de un día con lluvia tiene una variación estacional. Durante el verano se producen cambios graduales, aumentando el DDSLR continuamente hasta el final de septiembre/principios de octubre, reduciendo la probabilidad de alcanzar el umbral de las lluvias. Sin embargo, en el sector central y oriental de las cordilleras béticas litorales la probabilidad de la lluvia disminuyó cuando el DRT aumentó, incluso en noviembre, con toda una serie de implicaciones sobre el sistema ecogeomorfológico. En el sector occidental, la probabilidad de que una precipitación supere el umbral de 10,0 mm fue notablemente superior y alcanzó el $90 \%$ en octubre. Sin embargo, la de precipitaciones reducidas $(0,1 \mathrm{~mm})$, durante el otoño era mayor en el extremo oriental, aunque las canti- 
dades eran insignificantes. En definitiva, a lo largo del sur de España, conforme baja el umbral (DRTs $=0,1-5,0 \mathrm{~mm}$ ) menores son las diferencias, mientras que en los DRTs elevados aparecen grandes diferencias en la probabilidad de lluvias (Ruiz Sinoga et al., 2012).

La distribución de las medias de DDLSR para varios DRTs permitió la comparación entre diferentes observatorios a lo largo del gradiente pluviométrico. Para DRT de 0,1 mm la evolución de las medias de DDSLR fue similar a lo largo del gradiente pluviométrico, sin embargo, para DRT superior a $0,1 \mathrm{~mm}$ las diferencias entre sitios fueron más evidentes, conforme aumentaban los valores de DDSLR y la aridez. La zona occidental tiene menos variabilidad temporal que la oriental. Por último, para DRT de 30,0 mm el DDSLR adquiere los mayores valores la zona oriental (GE con 220 días), con más de 7 meses.

La variabilidad espacial y temporal del número de días sin lluvias, o días de lluvia con diferentes umbrales, es una característica climática propia del gradiente pluviométrico analizado. En los observatorios más occidentales hay una marcada y significativa disminución del número de días con precipitación superior a $10 \mathrm{~mm}$, sin embargo, en los restantes observatorios hay un aumento significativo del número de días con más de $30 \mathrm{~mm}$ de precipitación. Estos eventos de lluvias intensas son comunes en el final del invierno y el comienzo del otoño y tienen un efecto marcado sobre la erosión del suelo debido a que en esa época la cobertura vegetal es escasa o inexistente. Sin embargo, en los orientales, mientras se reducen los días con precipitaciones no intensas, se incrementan los eventos torrenciales $(\geq 30 \mathrm{~mm})$, lo que se traduce en un incremento de la aridez, una reducción de la cubierta vegetal y el inicio de los procesos de desertificación (Tabla 4).

Tabla 4

ANÁLISIS DESCRIPTIVO DEL NÚMERO DÍAS SIN LLUVIA Y DE LAS SECUENCIAS DE SEQUÍA. PERIODO DE ANÁLISIS: 1964-2008

\begin{tabular}{|c|c|c|c|c|}
\hline Observatorio & $\begin{array}{c}\text { Días sin lluvia } \\
(\%)\end{array}$ & $\begin{array}{c}\mathrm{N}^{o} \text { de secuencias } \\
\text { de sequía }\end{array}$ & $\begin{array}{l}\text { Secuencia de } \\
\text { sequía media } \\
\text { (días) }\end{array}$ & $\begin{array}{l}\text { Secuencia de } \\
\text { sequía máxima } \\
\text { (días) }\end{array}$ \\
\hline GA & 78,1 & 1193 & 10,9 & 161 \\
\hline $\mathrm{LC}$ & 84,3 & 1260 & 11,2 & 156 \\
\hline $\mathrm{AM}$ & 87,6 & 1117 & 13,1 & 198 \\
\hline GE & 92,4 & 872 & 17,8 & 489 \\
\hline
\end{tabular}

\section{IV.3. La dinámica eco-geomorfológica}

La existencia de un gradiente pluviométrico, como el analizado, lleva implícito una variada distribución espacial del agua disponible por parte del sistema eco-geomorfológico, que puede traducirse en diferentes procesos dentro de las relaciones suelo-agua-planta. De ahí la necesidad de llevar a cabo un análisis exhaustivo de las principales propiedades físicas, químicas e hidrológicas de los suelos sobre los que se sustentan los ecosistemas mediterráneos, al objeto de determinar los factores que inciden en el control de los procesos geomórficos. Como ya ha sido reseñado, y dada la cantidad de muestras y variables analizadas, se han 
utilizado diversos estadísticos al respecto. Inicialmente, y con el fin de determinar la relación entre todas las variables seleccionadas se realizó una matriz de correlación de Pearson (Tabla 5). Los resultados obtenidos mostraron una muy alta significación estadística entre la mayoría de las variables analizadas.

En términos generales se observó como un incremento de la pluviometría a lo largo del gradiente, hacía aumentar otras variables como: la humedad del suelo (media y máxima), la cobertura vegetal, la biodiversidad o número de especies vegetales, el contenido de materia orgánica de los suelos, la cantidad de carbono orgánico retenido, la capacidad de intercambio catiónico, y la conductividad hidráulica. Y por el contrario, se producía una reducción de la erodibilidad del suelo (factor K). Asimismo, las propiedades que ofrecían una correlación más elevada y significativa entre ellas fueron el contenido de materia orgánica, la cantidad de carbono orgánico retenido, la capacidad de intercambio catiónico y el factor $\mathrm{K}$.

Esto coincide con lo planteado por diversos autores en relación al papel que ejerce un gradiente pluviométrico sobre el sistema eco-geomorfológico en condiciones mediterráneas (Lavee et al., 1998), y se corrobora tras el análisis factorial realizado mediante ACP, cuyo KMO test puede considerarse de excelente $(0,864)$. El ACP mostró 4 factores que explican el 76,5\% de la varianza, con una marcada polarización (Tabla 6). El primer factor (F1) es el de más peso estadístico y explica el 54,7\%, siendo relativo a la incidencia del gradiente pluviométrico en los factores eco-geomorfológicos del sistema, y así, se asocia con la cubierta vegetal, materia orgánica, carbono orgánico, capacidad de intercambio catiónico, conductividad hidráulica saturada, Factor K de la USLE, densidad aparente, y arenas muy finas. Es decir, se muestra como un incremento de la pluviometría incide en la estabilidad del sistema. O lo que es igual, como la disponibilidad de agua, garante de la pervivencia de la vegetación, se convierte en un factor estabilizador del sistema eco-geomorfológico. Lo que, en sí mismo, ya es un factor importante al considerar la dinámica a la que pueden verse sometidos los distintos ambientes mediterráneos en el contexto de los escenarios barajados para el mediterráneo occidental por el IPCC.

Los restantes factores tienen bastante menor peso estadístico y sobretodo muestran una vinculación directa con el anterior. Así, F2 (9,9\% de la varianza) es relativo a la capacidad de transferencia hidrológica del suelo, humedad del suelo, con SMin and SMax, lo que evidencia la dinámica hidrológica de su perfil. F3 (6,5\% de la varianza) se refiere al componente estructural del suelo o su resistencia a ser destruido o degradado por procesos como el impacto de las gotas de lluvia o la escorrentía superficial, relacionando la estabilidad estructural con el contenido en arcillas y la biodiversidad. Por último, F4 (5,3\% de la varianza) hace referencia al componente textural del suelo, puesto que vincula un incremento del contenido de limos con una reducción del de arenas y viceversa.

Los resultados obtenidos del ACP confirman que el uso de la aproximación desde el gradiente pluviométrico al análisis del estado de salud del sistema eco-geomorfológico en condiciones mediterráneas es un buen método para caracterizar y determinar procesos de degradación del suelo. En efecto, se ha constatado una degradación del suelo y de sus principales propiedades indicadoras desde las áreas mas húmedas a las áridas (Ruiz Sinoga y Romero Díaz, 2010). Sin embargo, el peso de los distintos factores en el sistema varía a lo largo del gradiente considerado en cada una de las áreas de estudio. 
$\Xi \mathbb{1}$

心㟔胥或

कर

战出

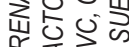

崖㱏空㟧

㟔で出口

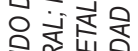

出岩

岁论岁

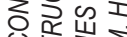

U足剀

प्ये

东出

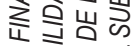

ํㅜㄴ엄

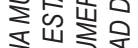

空的胥

究

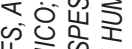

岁等

نं

这这乐

璐之足

这安

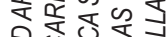

전

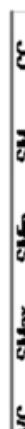

\&

중

5

紊

\

$\frac{5}{5}$

$\underline{x}$

שु

之언

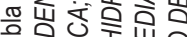

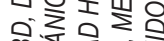

멍준

낭

之衣包

岃司崫

징

넘

응

넌전

这足崖

닐

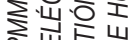

때임

추원

웅올

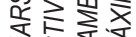

떵엉

崖罂

口产5

之언

0心㟧

히는

옹은전

O전

岂㟔空क

수었

仚出的出

点嵌

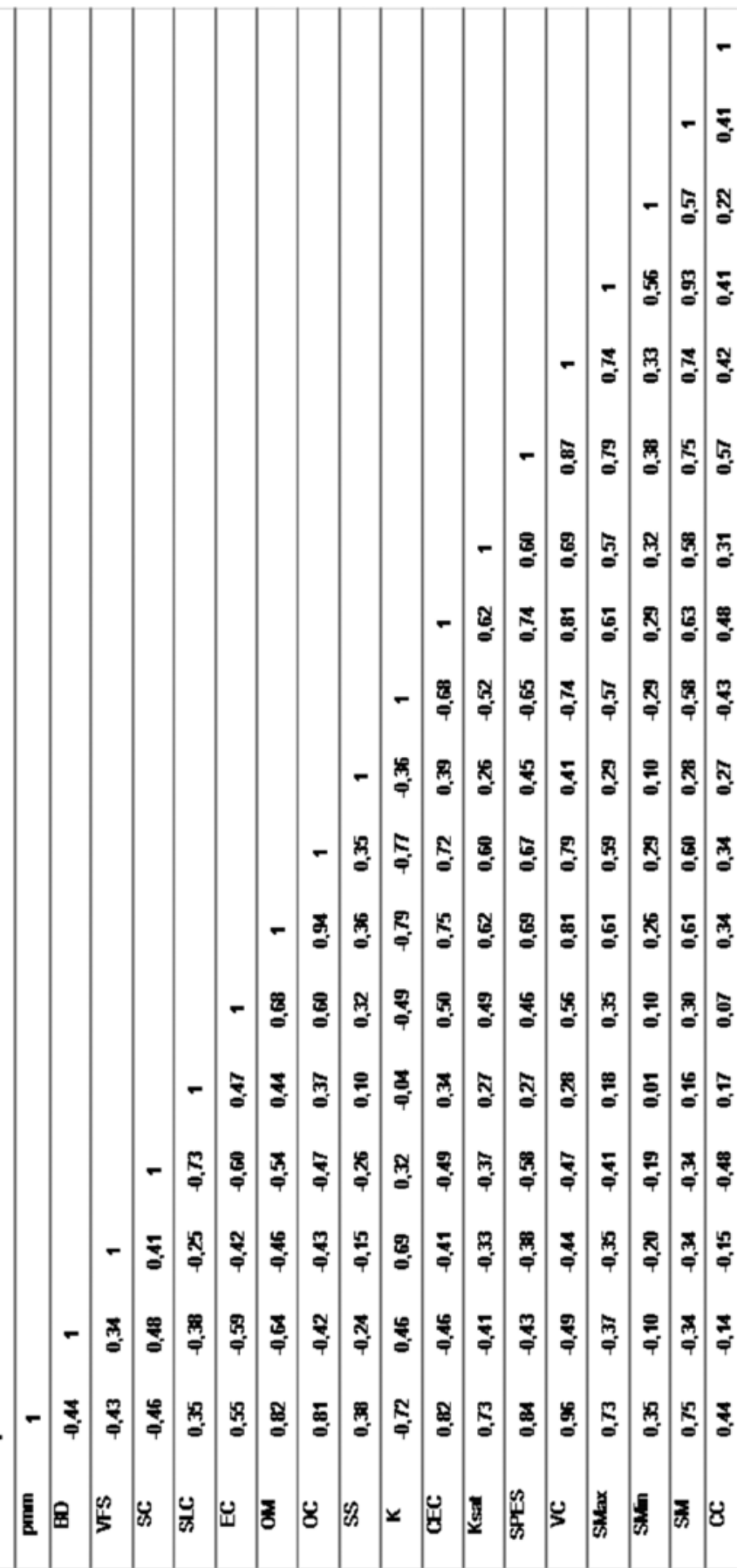

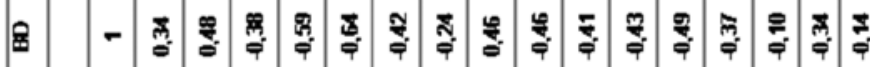

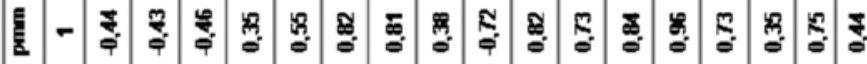

E 
Tabla 6

ANÁLISIS FACTORIAL (COMPONENTES PRINCIPALES). VARIABLES: BD, DENSIDAD APARENTE; VFS, ARENA MUY FINA; EC, CONDUCTIVIDAD ELÉCTRICA; OM, MATERIA ORGÁNICA; OC, CARBÓN ORGÁNICO; K, FACTOR K DE LA USLE; CEC, CAPACIDAD DE INTERCAMBIO CATIÓNICO; KSAT, CONDUCTIVIDAD HIDRÁULICA SATURADA; VC, CUBIERTA VEGETAL; PMM, PRECIPITACIÓN EN MM.; SMAX, MEDIA DE LAS MÁXIMAS DE HUMEDAD DEL SUELO; SMIN, MEDIA DE LAS MÍNIMAS DE HUMEDAD DEL SUELO; SM, HUMEDAD DEL SUELO; CC, CONTENIDO DE ARCILLAS; SS, ESTABILIDAD ESTRUCTURAL; SPES, NUMERO DE ESPECIES VEGETALES; SC, CONTENIDO DE ARENA; SLC, CONTENIDO DE LIMOS

\begin{tabular}{lrrrr}
\hline \multicolumn{5}{c}{ Factor } \\
\hline & \multicolumn{1}{c}{ F1 } & \multicolumn{1}{c}{ F2 } & \multicolumn{1}{c}{ F3 } & \multicolumn{1}{c}{ F4 } \\
\hline BD & $-\mathbf{0 , 6 2 4}$ & $-0,050$ & $-0,029$ & $-0,388$ \\
\hline VFS & $-\mathbf{0 , 6 0 5}$ & $-0,196$ & 0,064 & $-0,135$ \\
\hline EC & $\mathbf{0 , 7 3 1}$ & $-0,017$ & 0,042 & 0,449 \\
\hline OM & 0,846 & 0,249 & 0,267 & 0,250 \\
\hline OC & 0,796 & 0,282 & 0,297 & 0,151 \\
\hline K & $-0,797$ & $-0,293$ & $-0,320$ & 0,124 \\
\hline CEC & 0,598 & 0,361 & 0,471 & 0,199 \\
\hline Ksat & 0,551 & 0,438 & 0,202 & 0,157 \\
\hline VC & 0,688 & 0,460 & 0,431 & 0,116 \\
\hline pmm & 0,668 & 0,483 & 0,417 & 0,152 \\
\hline SMax & 0,385 & 0,789 & 0,264 & 0,106 \\
\hline SMin & 0,070 & 0,824 & $-0,070$ & 0,012 \\
\hline SM & 0,388 & 0,804 & 0,265 & 0,047 \\
\hline CC & $-0,028$ & 0,350 & 0,733 & 0,238 \\
\hline SS & 0,282 & $-0,073$ & 0,705 & $-0,004$ \\
\hline SPES & 0,458 & 0,538 & 0,557 & 0,213 \\
\hline SC & $-0,271$ & $-0,189$ & $-0,282$ & $-0,833$ \\
\hline SLC & 0,221 & 0,006 & 0,026 & 0,896 \\
\hline
\end{tabular}

Tabla 7

VARIANZA EXPLICADA POR CADA FACTOR (\%) YACUMULADA (\%AC), PARA LAS DISTINTAS ÁREAS DE ESTUDIO

\begin{tabular}{|c|c|c|c|c|c|c|c|c|c|c|c|c|c|c|c|c|}
\hline \multirow[b]{2}{*}{ FACTOR } & \multicolumn{2}{|c|}{ GAUCIN } & \multicolumn{2}{|c|}{ MARBPIIA } & \multicolumn{2}{|c|}{ COIMENAR } & \multicolumn{2}{|c|}{ NMOGIA } & \multicolumn{2}{|c|}{ CAMPANIILAS } & \multicolumn{2}{|c|}{ BERIA } & \multicolumn{2}{|c|}{ NBUÑOL } & \multicolumn{2}{|c|}{ GERGN } \\
\hline & $\%$ & XAC & $\%$ & XAC & $\%$ & Xac & $\%$ & XAC & $\%$ & ×AC & $\%$ & XAC & $\%$ & XAC & $\%$ & *AC \\
\hline 1 & 31,91 & 31,91 & 35,65 & 35.65 & 31,30 & 34,30 & 36,08 & 36.08 & 22,33 & 22,33 & 20.50 & 29.50 & 8,68 & 25,68 & 29,97 & 29.97 \\
\hline 2 & 12,58 & 44,49 & 16,41 & 52,06 & 13,49 & 47,79 & 16,83 & 52,91 & 16,89 & 39,22 & 20,18 & 49,68 & 22,43 & 48,11 & 17,89 & 47,86 \\
\hline 3 & 9,44 & 53,93 & 14,65 & 66,71 & 10,57 & 58,36 & $8, \pi 2$ & 61,64 & 12,72 & 51,94 & 11,83 & 61,51 & $\mathbf{u , 0 3}$ & 62,15 & ห, 07 & 61,93 \\
\hline 4 & 8,05 & 61,98 & 7,36 & 74,07 & 8,17 & 66,53 & 7,55 & 69,19 & 10,87 & 62,81 & 9,80 & 71,31 & 7,요 & 69,73 & 8,00 & 69,93 \\
\hline 5 & 7,09 & 69,08 & 6,32 & 80,39 & 6,74 & 73,27 & & & 8,82 & 71,63 & & & 6,83 & 76,57 & 6,75 & 76,68 \\
\hline 6 & 6.36 & 75,43 & & & 6.50 & 79,76 & & & 6.60 & $\pi n$ & & & & & & \\
\hline
\end{tabular}

Los valores del índice KMO obtenidos, que mide la idoneidad de los datos para realizar un análisis factorial comparando los coeficientes de correlación, siempre estuvieron por encima de 0,752 , lo que muestra su alta significancia estadística, con unos elevados porcentajes de la varianza explicada, y un número de factores que oscila entre 4 y 6 , aunque en todos los casos hay un factor dominante (Tabla 7).

En términos generales, el patrón de degradación del suelo es diferente según el ámbito climático: en los ambientes más húmedos, la degradación parece estar controlada por la 
humedad del suelo asociada a las propiedades indicadoras de estabilidad, mientras que en los más secos es la ausencia de vegetación vinculada a los procesos de degradación del suelo. En cualquier caso, son los factores bióticos los que muestran la estabilidad o inestabilidad del sistema, así, la materia orgánica o el carbono orgánico retenido en el suelo son propiedades que están vinculadas a los factores más determinantes.

En ambientes mediterráneos húmedos (Gaucín), el factor relacionado con los procesos bióticos (F1), humedad del suelo, SMax, materia orgánica, carbono orgánico y su consecuencia directa en la reducción del factor K, es el más importante, explicando el 31,9\% de la varianza. Se trata de un sistema eco-geomorfológico estable, con suficientes aportes pluviométricos para garantizar una buena cubierta vegetal, e iniciar el ciclo del carbono orgánico. Sin embargo, la posible tendencia hacia la reducción pluviométrica (IPCC, 2013), sitúa a estos ambientes en uno de los escenarios de riesgo en los que la menor disponibilidad de agua por parte del ecosistema, contribuirá a su progresivo empobrecimiento y disminución de la importancia de los factores relativos al ciclo orgánico.

En ambientes mediterráneos sub-húmedos, el ACP muestra una polarización del primer factor sobre los demás, explicando en Marbella y Colmenar el 35,6\% y el 34,3\% de la varianza, respectivamente, y a pesar de la reducción de la cubierta vegetal, el F1 está asociado con los procesos bióticos, al relacionar el incremento de la humedad del suelo, con la cubierta vegetal, con el contenido de materia orgánica y la captura de carbono orgánico, y con la salinidad (en Marbella) o el factor K (en Colmenar). En estos ambientes, aunque hay un peso específico elevado, por parte de los factores bióticos, en la explicación del comportamiento del sistema eco-geomorfológico, los factores relacionados con las características edáficas empiezan a tener una significativa importancia. En estas zonas, y especialmente en la costa occidental, de continuar la tendencia actual hacia el incremento pluviométrico, puede situar a estos ambientes bajo unas condiciones de estabilidad, en donde los procesos bióticos seguirían controlando la dinámica del sistema, convirtiéndose en las únicas áreas estables del sur de España.

En ambientes mediterráneos secos, la reducción tanto del número de especies vegetales como de cubierta vegetal conduce a una mayor desprotección del suelo, que es mayor conforme menor es la pluviometría. Esto se traduce en una pérdida de peso de los procesos relacionados con los factores bióticos, y el incremento de aquellos vinculados a los procesos edáficos o abióticos. En Almogía, los factores relacionados con la cubierta vegetal (F1) explican el 36,0\% de la varianza, y vinculan las áreas con una mayor humedad, tanto en valores medios como máximos, con una mayor cubierta vegetal, contenido de materia orgánica, captura de carbono orgánico, capacidad de intercambio catiónico, e inversamente con el factor K. Es decir, es el factor de estabilidad del sistema. Los restantes factores están relacionados con las variables abióticas del sistema. Así pues, aparecen unos claros síntomas de degradación que se agudizan conforme las condiciones ambientales muestran rasgos semiáridos, bajo las que el sistema eco-geomorfológico empieza a estar estructurado por los factores abióticos. Estos ambientes, de continuar la tendencia pluviométrica observada durante el último medio siglo, serán los que de una forma más drástica padezcan la reducción en cuanto a disponibilidad de recursos hídricos. Si actualmente se sitúan en el umbral entre los factores bióticos y los abióticos, la reducción de aportes de lluvia puede conducirlos hacia procesos más típicos de regiones mediterráneas semiáridas. 
En ambientes semiáridos, el ACP muestra como los factores bióticos son responsables del 29,5\% de la varianza, y los abióticos del 41,81\% (Berja), mientras que dichas cifras son del 22,4\% y 51,0\% respectivamente en Albuñol. Así en Berja, el principal factor (F1) está relacionado directamente con el ciclo de la materia orgánica derivado de la cubierta vegetal (explicando el $29,5 \%$ de la varianza), asociado a su vez a un incremento de la salinidad del suelo, propio de condiciones semiáridas, y a unos inferiores valores de densidad aparente. Y en Albuñol, el primer factor (F1) que explica el 25,6\% de la varianza, vincula las condiciones hidrológicas del suelo, mediante su contenido de humedad, a la existencia de una menor erodibilidad $(\mathrm{K})$ y cantidad de arenas muy finas, siendo las áreas cuya humedad del suelo son más elevadas, aquellas que poseen valores inferiores de la fracción granulométrica más susceptible de ser transportada, así como del factor K. Así pues, conforme se reduce la pluviometría, los indicadores de degradación se vinculan a la existencia de un mayor o menor contenido de humedad del suelo, sin que exista una relación entre la humedad y la cubierta vegetal, manifestada a través del ciclo de la materia orgánica. Estos ambientes, ante la actual tendencia hacia la reducción pluviométrica, se verán abocados a procesos de degradación del suelo y desertificación, en los que serán los factores abióticos los que controlen la degradación del sistema eco-geomorfológico, hacia condiciones de aridez.

Por último, en el ambiente más árido analizado (Gérgal), el primer factor (F1) explica el 29,9\% de la varianza y vincula la vegetación, a través del contenido de OM y captura de OC, con áreas de mayor salinidad y menor densidad aparente, circunstancias que coinciden en las tres áreas de estudio más secas. En estas áreas, la existencia de un mayor contenido de humedad obedece a una mayor Ksat, como respuesta inmediata a la precipitación, y este proceso solo se produce allí donde el contenido de arcillas es bajo. Por el contrario, fracciones de arcilla elevadas están relacionadas con procesos de degradación del suelo por erodibilidad. Estos factores abióticos explican el 46,7\% de la varianza. Sin embargo, la existencia de unos mayores niveles de materia orgánica y por tanto de vegetación, no están vinculados como en los ambientes húmedos con un incremento de la humedad del suelo, sino con una menor densidad aparente y unos mayores contenidos de salinidad, como máxima expresión de degradación del sistema. Si estos ambientes en la actualidad regulan muy poca agua $(<$ $250 \mathrm{~mm} / \mathrm{año}$ ), la tendencia hacia la reducción pluviométrica los situara en unas condiciones de aridez extrema, en las que los procesos de desertificación alcanzaran un grado máximo de irreversibilidad.

\section{CONCLUSIONES}

La preocupación sobre los escenarios de escasez de recursos hídricos en el contexto del Cambio Climático y sus implicaciones eco-geomorfológicas en las Cordilleras Béticas Litorales, ha mostrado la necesidad de llevar a cabo un análisis crítico de la evolución temporal de la precipitación en la zona, con el fin de establecer si se está produciendo una reducción progresiva.

1. Los resultados muestran la existencia de un gradiente pluviométrico desde la zona occidental a la oriental, y marcados contrastes tanto longitudinales como latitudinales, y así, mientras en la costa sureste se observó una disminución en la precipitación, en la costa suroeste la tendencia ha sido la contraria, contrastando con los escenarios que se han divul- 
gado derivados del uso de ciertos modelos predictivos. En las áreas interiores de las Cordilleras Béticas Litorales, las tendencias de disminución de la precipitación son evidentes, en especial en invierno y otoño.

2. El índice DDSLR aumenta en proporción a la aridez, lo cual es lógico y, por tanto, la vegetación ha de adaptarse a tales condiciones xéricas. Sin embargo, hay poca variación a lo largo del gradiente pluviométrico con respecto a los valores DDSLR entre lluvias de escasa cantidad (inferiores a $1 \mathrm{~mm} /$ día), y grandes diferencias entre lluvias de cierta entidad (entre 10,0-30,0 mm/día). En los ambientes orientales se incrementa el número de días sin precipitación, pero también sufren un aumento de la probabilidad de precipitaciones extremas superiores a $30 \mathrm{~mm}$ día.

3. A lo largo de un gradiente pluviométrico mediterráneo, desde condiciones húmedas a semiáridas-áridas, la relación de los factores bióticos/abióticos controlan los procesos de degradación del suelo. Dependiendo del factor dominante y de la dinámica pluviométrica se modificará el proceso de formación/degradación del suelo.

4. Conforme se reduce la pluviometría, los indicadores de degradación del suelo se vinculan a una mayor o menor humedad en el mismo, sin que exista vinculación entre el contenido de agua en el suelo y la cubierta vegetal, manifestada a través del ciclo de la materia orgánica. En los ambientes mediterráneos húmedos los procesos de formación del suelo están vinculados a una mayor pluviometría, que incide en el porcentaje de cubierta vegetal, en el contenido de humedad del suelo, y en el incremento de la fracción orgánica, suponiendo un proceso de retroalimentación positivo. Por el contrario, en ambientes mediterráneos semiáridos-áridos, la existencia de cubierta vegetal, solo está vinculada a factores estructurales del suelo como la densidad aparente, y los mayores o menores contenidos de humedad del suelo se relacionaran directamente con factores texturales como respuesta inmediata de las escasas precipitaciones.

5. Este proceso de degradación es lineal, situándose el umbral de degradación del suelo entorno a los $500 \mathrm{~mm} /$ año, puesto que es a partir de ahí, cuando la vegetación no está asociada a la existencia de mayores contenidos de humedad en el suelo, adaptándose a la degradación del mismo, como puede comprobarse mediante la aparición de las especies xerófitas.

6. De continuar la actual tendencia pluviométrica, una gran parte del sistema eco-geomorfológico del sur de España, se situaría por debajo de ese umbral de degradación del suelo, con la consiguiente evolución hacia escenarios áridos.

7. El método para abordar el estudio de los procesos de degradación del suelo, mediante la aproximación a partir de la definición de gradientes pluviométricos y el análisis de la tendencia de la pluviometría, así como las propiedades del suelo consideradas, es adecuado para la determinación de los factores incidentes en los mismos. Para ello, se ha mostrado muy satisfactoria la aplicación del método de análisis de componentes principales.

\section{REFERENCIAS}

AFNOR (1987): Qualité de sols, methods d'analyse. AFNOR (Association française de normalisation). Paris, France.

AGUILAR, M., SÁNCHEZ RODRÍGUEZ, E. y PITA, M.F. (2006): «Tendencia de las precipitaciones de marzo en el sur de la Península Ibérica», In Clima, Sociedad y Medio Ambiente, Asociación Española de Climatología, Zaragoza, 41-51. 
ALEXANDERSSON, H. (1986): «A Homogeneity Test Applied to Precipitation Data». Journal of Climatology, 6, 661-675.

ALEXANDERSSON, H. y MOBERG, A. (1997): «Homogeneization of Swedish Temperature Data. Part I: Homogeneity Test for Linear Trends». Internacional Journal of Climatology, 17, 25-34.

ARONSON, J. y SHMIDA, A. (1992): «Plant species diversity along a mediterranean-desert gradient and its correlation with interannual rainfall fluctuations». Journal of Arid Environments, 23, 235-247.

AVIAD, Y., KUTIEL, H. y LAVEE H. (2009): «Variation of Dry Days Since Last Rain (DDSLR) as a measure of dryness along a Mediterranean-Arid transect». Journal of Arid Environments 73, $658-665$.

BALLIETTE, J.F., MCDANIEL, K.C. y WOOD, M.K., (1986): «Infiltration and sediment production following chemical control of Sagebrush in New Mexico». J. Range Manage. 39, 160-165.

BEATLEY J.C. (1974): «Phenological events and their environmental triggers in Mojave desert ecosystems». Ecology, 55: 856 -863.

BERGER A. y GOOSSENS C. (1983): «Persistence of wet and dry spells at Uccle (Belgium)». Journal of Climatology, 3, 21-34.

BERGKAMP, G., CERDÀ, A. e IMESON, A.C. (1999): «Magnitude-frequency analysis of water redistribution along a climate gradient in Spain». Catena, 37 (1-2), 129-146.

BLAKE, G.R. y HARTGE, K.H. (1986): «Bulk density». In: Klute, A. (Ed.), Methods of Soil Analysis, Part 1, $2^{\text {nd }}$ Edition. Agronomy Monograph, Vol. 9. American Society of Agronomy, Madison, WI, pp. 363-375.

BOWER, C.A., REITMEIR, R.F. y FIREMAN, M. (1952): «Exchangeable cation analysis of saline and alkali soils». Soil Sci. 73, 251-261.

BOWERS, M.A. (1987): «Precipitation and the relative abundance of desert winter annuals: A 6-year study in the northern Mojave Desert». Journal of Arid Environments, 12, 141-149.

BRIGHTMAN, H.J. (1999): Data Analysis In Plain English with Microsoft Excel, 1st Edition, Georgia State University, 594 pp.

BRUNET, M., CASADO, M., CASTRO, M., GALÁN, P., LÓPEZ, J., MARTÍN, J., PASTOR, A., PETISCO, E., RAMOS, P., RIBALAYGUA, J., RODRÍGUEZ, E., SANZ, I. y TORRES, L. (2008): Generación de escenarios regionalizados de cambio climático para España. Technical report, Agencia Estatal de Meteorología (AEMET), Ministerio de Medio Ambiente y Medio Rural y Marino.

CAMMERAAT, L.H. y IMESON, A.C. (1998): «Deriving indicators of soil degradation from soil aggregation studies in southeastern Spain and southern France». Geomorphology, 23, 307-321.

CASTILLO, J.M. (1989): El clima de Andalucía, Almería, Instituto de Estudios Almerienses.

CAPEL, J.J. (1987): «El clima de Andalucía». In Cano, G. (Ed.): Geografía de Andalucía, Sevilla, Ed. Tartessos, Vol. II, 99-186.

CEBALLOS, A. y SCHNABEL, S. (1998): «Hydrological behaviour of a small catchment in the dehesa land use system (Extremadura, SW Spain)». Journal of Hydrology, 210, 146-160. 
CEBALLOS, A., MARTÍNEZ FERNÁNDEZ, J., SANTOS, F. y ALONSO, P. (2002): «Soil - water behaviour of sandy soils under semi-arid conditions in the Duero Basin (Spain)». Journal of Arid Environments, 51, 501-519.

CEBALLOS, A., MARTÍNEZ FERNÁNDEZ, J. y LUENGO-UGIDOS, M. A. (2004): «Analysis of rainfall trends and dry periods on a pluviometric gradient representative of Mediterranean climate in the Duero Basin, Spain». Journal of Arid Environments, 58, 215-233.

CERDÁ, A. (1998): «Relationships between climate and soil hydrological and erosional characteristics along climatic gradients in Mediterranean limestone areas». Geomorphology, $25,123-134$.

COOK, G.D. y HEERDEGEN, R.G. (2001): «Spatial variation in the duration of the rainy season in monsoonal Australia». International Journal of Climatology, 21, 1.723-1.732.

DÍAZ, H.F., BRADLEY, R.S. y EISCHEID, J.K. (1989): «Precipitation fluctuation over global land areas since the late 1800's». Journal of Geophysical Research, 94, 1095-1120.

DE CASTRO, M., MARTÍN VIDE, J. y ALONSO, S. (2005): «El clima de España: pasado, presente y escenarios de clima para el siglo XXI». In Moreno, J.M. (Ed.): Evaluación Preliminar de los Impactos en España por Efecto del Cambio Climático. Ministerio de Medio Ambiente, Madrid, pp. 1-64.

DE LUIS, M., GONZALEZ HIDALGO, J.C., LONGARES, L.A. y STEPÁNEK, P. (2009): «Seasonal precipitation trends in the Mediterranean Iberian Peninsula in second half of 20th century». International Journal of Climatology, 29, 1.312-1.323.

DOUGUEDROIT, A. (1987): «The variation of dry spells in Marseilles from 1865 to 1984». Journal of Climatology 7: 541-551.

DUNNE, T., ZHANG, W. y AUBRY, B.F. (1991): «Effects of rainfall, vegetation and microtopography on infiltration and runoff». Water Resources Research, 27, 2.271-2.285.

ESTEBAN PARRA, M.J., POZO VAZQUEZ, D., RODRIGO, F.S. y CASTRO DIEZ, Y. (2003): «Temperature and precipitation variability and trends in Northern Spain in the context of the Iberian peninsula climate». In: Bölle H-J (Ed) Mediterranean climate. Variability and trends. Springer, 259-276.

GHAZANFAR, S.A. (1991): «Vegetation structure and phytogeography of Jabal Sham, an arid mountain in Oman». Journal of Biogeography, 18, 299-309.

GIORGI, F. y LIONELLO, P. (2008): «Climate change projections for the Mediterranean region». Global. Planet. Change, 63, 90-104.

GONZÁlEZ HIDAlGO, J.C., DE LUÍS, M., RAVENTOS, J. y SÁNCHEZ, J. (2001): «Spatial distribution of seasonal rainfall trends in a western mediterranean area». International journal of climatology, 21 (7), 843-860.

GONZALEZ HIDALGO, J.C., LÓPEZ BUSTINS, J.A., STEPÁNEK, P., MARTÍN VIDE, J. y DE LUIS, M. (2009): «Monthly precipitation trends on the Mediterranean fringe of the Iberian Peninsula during the second half of the 20th century (1951-2000)». International Journal of Climatology 29, 1.415-1.429.

GUIJARRO, J.A. (2002): «Tendencias de la precipitación n en el litoral Mediterráneo español». Proceedings of III Congreso de la Asociación Española de Climatología, L'aigua en el clima, Palma de Mallorca, 237-246.

HELD, I.M. y SODEN, B.J. (2006): «Robust responses of the hydrological cycle to global warming». J. Clim., 19, 5686-5699. 
HULME, M. (1995): «Estimating global changes in precipitation». Weather, 50, 34-42.

IMESON, A.C. y LAVEE, H. (1998): «Soil erosion and climate change: the transect approach and the influence of scale». Geomorphology, 23 (2-4), 219-227.

IPCC (2007): «Climate change 2007: the physical science basis». Contribution of Working Group I to the Fourth Assessment Report of the Intergovernmental Panel on Climate Change (Ed. by S. Solomon, D. Qin, M. Manning, Z. Chen, M. Marquis, K.B. Averyt, M. Tignor and H.L. Miller). Cambridge University Press, Cambridge.

IPCC (2013): «Climate Change 2013: The Physical Science Basis». Contribution of Working Group I to the Fifth Assessment Report of the Intergovernmental Panel on Climate Change [Stocker, T.F., D. Qin, G.-K. Plattner, M. Tignor, S.K. Allen, J. Boschung, A. Nauels, Y. Xia, V. Bex and P.M. Midgley (eds.)]. Cambridge University Press, Cambridge, United Kingdom and New York, NY, USA, 1.535 pp.

JACKSON I. J. (1981): «Dependence of wet and dry days in the tropics». Archives for Meteorology, Geophysics and Bioclimatology, Series B 29, 167-179.

KUTIEL, P., LAVEE, H. y SHOSHANY, M. (1995): «Influence of a climatic gradient upon vegetation dynamics along a Mediterranean-arid transect». Journal of Biogeography, 22, 1.064-1.071.

KUTIEL, P., KUTIEL, H. y LAVEE, H. (2000): «Vegetation response to possible scenarios of rainfall variations along a Mediterranean-extreme arid climatic transect «. Journal of Arid Environments, 44, 277-290.

LAVEE, H., IMESON, A. C. y SARAH P. (1998): «The impact of climate change on geomorphology and desertification along a Mediterranean arid transect». Land Degradation and Development, 9, 407-422.

LE BISSONNAIS, Y. (1996): «Aggregate stability and assessment of soil crustability and erodibility: I. Theory and methodology». European Journal of Soil Science, 47, 425-437.

LE HOUEROU, H.N., BINGHAM, R.L. y SKERBEK, W. (1988): «Relationship between the variability of primary production and the variability of annual precipitation in world arid lands». J. Arid Env. 15, 1-18.

LÓPEZ BUSTINS, J.A., MARTÍN VIDE, J. y SÁNCHEZ LORENZO, A. (2008a): «Iberian winter rainfall trends based upon changes in teleconnection and circulation patterns». Global and Planetary Change, 63 (2-3), 171-176.

LÓPEZ BUSTINS, J.A., SÁNCHEZ LORENZO, A., AZORÍN MOLINA, C. y ORDÓÑEZ LÓPEZ, A. (2008b): «Tendencias de la precipitación invernal en la fachada oriental de la Península Ibérica». In: Sigró, J., Brunet, M. and Aguilar, E. (Eds.): Cambio Climático Regional y sus Impactos, Asociación Española de Climatología, Serie A, $\mathrm{n}^{\circ} 6$, 161-171.

MEEHL, G. A (2007). «Global climate projections». In: Climate Change 2007: The Physical Science Basis. Contribution of Working Group I to the Fourth Assessment Report of the Intergovernmental Panel on Climate Change [Solomon, S., D. Qin, M. Manning, Z. Chen, M. Marquis, K. B. Averyt, M. Tignor and H. L. Miller (eds.)] Cambridge University Press, Cambridge, United Kingdom and New York, NY, USA, pp. 747-846.

MEHTA, A.V. y YANG, S. (2008): «Precipitation climatology over Mediterranean Basin from ten years of TRMM measurements». Advances in Geosciences, 17, 87-91. 
MORENO GARCÍA, M.C. y MARTÍN VIDE, J. (1986): «Estudio preliminar sobre las tendencias de la precipitación anual en el sur de la Península Ibérica: el caso de Gibraltar». II Simposio del Agua en Andalucía, Dpto. Hidrogeología. Universidad de Granada, I, 37-44.

MARTÍN VIDE, J. y FERNÁNDEZ, D. (2001). «El índice NAO y la precipitación mensual en la España peninsular». Investigaciones Geográficas, 26, 41-58.

MARTÍN VIDE J. y GOMEZ L. (1999): «Regionalization of peninsular Spain based on the length of dry spells». International Journal of Climatology, 19, 537-555.

MARTÍN VIDE, J. y LÓPEZ BUSTINS, J.A. (2006): «The Western Mediterranean Oscillation and rainfall in the Iberian Peninsula». International Journal of Climatology, 26 (11), $1.455-1.475$.

MARTÍNEZ MURILLO J.F. y RUIZ SINOGA J.D. (2007): «Seasonal changes in the hydrological and erosional response of a hillslope under dry-Mediterranean climatic conditions (Montes de Málaga, South of Spain)». Geomorphology, 88, 69 -83.

MAUGERI, M., BRUNETTI, M., MONTI, F. y NANNI, T. (2004): «Sea-Level pressure variability in the Po plain (1765-2000) from homogenized daily secular records «. International Journal of Climatology, 24, 437-455.

NORRANT, C., DOUGUÉDROIT, A. (2005): «Monthly and daily precipitation trends in the Mediterranean (1950-2000)». Theoretical and Applied Climatology, 83 (1-4), 89-106.

NEW, M., HULME, M. y JONES, P. (1999): «Representing twentieth-century space-time climate variability. Part I: Development of a 1961-90 mean monthly terrestrial climatology». J. Climate, 12, 829-856.

NOY MEIR, I. (1973): «Desert ecosystems: environment and producers». Annu. Rev. Ecol. Syst. 4, 25-51.

OECC y MMA. (2005): Principales conclusiones de la evaluación preliminar de los impactos en España por efecto del Cambio Climático. Proyecto ECCE, Ministerio de Medio Ambiente y Universidad de Castilla-La Mancha. Centro de Publicaciones de la Secretaría General Técnica (MMA).

PERZYNA, G. (1994): «Spatial and temporal characteristic of maximum dry spells in southern Norway». International Journal of Climatology, 14: 895-909.

PITA, M.F. (2003): «El clima de Andalucía». In: López, A. (Coord.): Geografía de Andalucía. Barcelona, Ariel, 137-174.

QUEREDA, J., MONTÓN, E. y ESCRIG, J. (2000): «La evolución de las precipitaciones en la Cuenca Occidental del Mediterráneo: ¿Tendencia o ciclos?». Investigaciones Geográficas, 24, 17-35.

REISER, H. y KUTIEL, H. (2010): «Rainfall uncertainty in the Mediterranean: dryness distribution». Theoretical and Applied Climatology, 100, 123-135.

ROBINSON (1922): «A new method for mechanical analysis of soil and other dispersion». J. Agr. Ac. 12, 306-321.

RUIZ SINOGA, J.D. y MARTÍNEZ MURILLO, J.F. (2009): «Eco-geomorphological system response variability to the 2004-06 drought along a climatic gradient of the Littoral Betic Range (southern Spain)». Geomorphology, 103, 351-362.

RUIZ SINOGA, J.D. y ROMERO DIAZ, A. (2010): Soil degradation factors along a Mediterranean pluviometric gradient in Southern Spain. Geomorphology, 118, 359-368. 
RUIZ SINOGA, J. D., GARCÍA MARÍN, R., MARTÍNEZ MURILLO, J. F. y GABARRON GALEOTE, M. A. (2011): «Precipitation dynamics in southern Spain: trends and cycles. International Journal of Climatology», 31, 2.281-2.289.

RUIZ SINOGA, J. D., GARCIA MARIN, R., GABARRON GALEOTE, M. A. y MARTINEZ MURILLO, J. F. (2012): «Analysis of dry periods along a pluviometric gradient in Mediterranean southern Spain». International Journal of Climatology, 32, 15581571.

SALADIÉ, O., BRUNET, M., AGUILAR, E., SIGRÓ, J. y LÓPEZ, D. (2002): «Evolución de la precipitación en el sector suroriental de la depresión del Ebro durante la segunda mitad del siglo XX». En Guijarro, J. A. Grimalt, M., Laita, M., Alonso, S. (Eds) El agua y el clima, Publicaciones de la AEC, Ser A, 3, 335-346.

SÁNCHEZ, E. y MIGUEZ-MACHO, G. (2010): «Proyecciones regionales de clima sobre la Península Ibérica: modelización de escenarios de cambio climático». In: Pérez F. Fiz y Boscolo Roberta (Eds.) Clima en España: pasado, presente y futuro, 69-80 pp.

SHOSHANY, M., KUTIEL, P., LAVEE, H. y EICHLER, M., (1994): «Remote sensing of vegetation cover along a climatological gradient». ISPRS J. Photogramm. and Remote Sensing, 49, 2-10.

SHOSHANY, M., LAVEE, H. y KUTIEL, P. (1995): «Seasonal vegetation cover changes as indicators of soil types along a climatological gradient: a mutual study of environmental patterns and controls using remote sensing». Int. J. Remote Sensing, 16, 2.137-2.151.

STEFANICKI, G., TALKNER, P. y WEBER, R. O. (1998): «Frequency Changes of Weather Types in the Alpine Region since 1945». Theoretical and Applied Climatology, 60, 47-61.

SUMNER, G.N., ROMERO, R., HOMAR, V., RAMIS, C., ALONSO y ZORITA, E. (2003): «An estimate of the effects of climate change on the rainfall of Mediterranean Spain by the late $21^{\text {st }}$ century». Climate Dynamics, 20, 789-805.

THUROW, T.L., BLACKBURN, W.H. y TAYLOR Jr., C.A. (1986): «Hydrologic characteristics of vegetation types as affected by livestock grazing systems, Edwards Plateau, Texas». J. Range Manage. 39, 505-509.

TONGWAY, D.J. y LUDWIG, J. A. (2003): «The nature of landscape dysfunction in rangelands». In: Ludwig, J.A., Tongway, D.J., Freudenberger, D., Noble, J., Hodgkinson, K. (Eds.), Landscape ecology function and management. CSIRO Publishing, Canberra, pp. 49-61.

TRIGO, I. F. (2006): «Climatology and interannual variability of storm-tracks in the EuroAtlantic sector: a comparison between ERA-40 and NCEP/NCAR reanalyses». Climate Dynamics, 26, 127-143.

VEENENDAAL, E.M., ERNST, W.H.O. y MODISE, G.S. (1996): «Effect of seasonal rainfall pattern on seedling emergence and establishment of grasses in a savanna in southeastern Botswana». Journal of Arid Environment, 32, 305-317.

WALFORD, N. (1995): Geographical Data Analysis. Wiley, 458 pp.

WARD, D. y OLSVIG-WHITTAKER, L. (1993): «Plant species diversity at the junction of two desert biogeographic zones». Biodiversity Letters, 1, 172-185.

WARREN, S. D., BLACKBURN, W. H. y TAYLOR Jr., C. A. (1986): «Effects of season and stage of rotation cycle on hydrologic condition of rangeland under intensive rotation grazing». J. Range Manage. 39, 486-491. 
WHEELER, D. y MARTÍN-VIDE, J. (1992): «Rainfall Characteristics of Mainland Europe's Most Southerly Stations». International Journal of Climatology, 12, 69-76.

WHITTAKER, R. H. (1975): Communities and Ecosystems. New York: MacMillan Press. $385 \mathrm{pp}$.

WISCHMEIER, W. H. y SMITH, D. D. (1978): «Prediction of rainfall splash erosion losses a guide to conservation planning». Agriculture Handbook No. 537. US Department of Agriculture, Washington, DC, $58 \mathrm{pp}$.

YAIR, A. y LAVEE, H. (1985): «Runoff generation in arid and semi-arid zones». In Hydrological Forecasting, Anderson MG, Burt TP (Eds), John Wiley and Sons: New York; pp. 183-220. 